\title{
WEIGHTED MATRIX EIGENVALUE BOUNDS ON THE INDEPENDENCE NUMBER OF A GRAPH*
}

\author{
RANDALL J. ELZINGA ${ }^{\dagger}$ AND DAVID A. GREGORY‡
}

\begin{abstract}
Weighted generalizations of Hoffman's ratio bound on the independence number of a regular graph are surveyed. Several known bounds are reviewed as special cases of modest extensions. Comparisons are made with the Shannon capacity $\Theta$, Lovász' parameter $\vartheta$, Schrijver's parameter $\vartheta^{\prime}$, and the ultimate independence ratio for categorical products. The survey concludes with some observations on graphs that attain a weighted version of a bound of Cvetković.
\end{abstract}

Key words. Independence number, Eigenvalues, Ratio bound, Graph, Matrix.

AMS subject classifications. 05C50, 05E99, 15A18.

1. Introduction. Throughout the survey, $G$ is assumed to be a simple graph with vertex set $V=\{1,2, \ldots, n\}$ and at least one edge. The adjacency matrix of $G$ will be denoted by $A=A(G)$. Thus $A_{i, j}=1$ if $i j$ is an edge of $G$ and $A_{i, j}=0$ otherwise. A weight matrix for $G$ is a real symmetric $n \times n$ matrix $W$ with zero diagonal such that $W_{i, j}=0$ whenever $i j$ is not an edge of $G$ and $W_{i, j} \neq 0$ for at least one edge $i j$ of $G$. Thus a weight matrix may have negative entries. Following Luz [20], a real symmetric $n \times n$ matrix $W$ with zero diagonal will be called an extended weight matrix for $G$ if $W_{i, j} \leq 0$ whenever $i j$ is not an edge of $G$ and $W_{i, j} \neq 0$ for at least one edge $i j$ of $G$. Thus, every weight matrix is an example of an extended weight matrix.

The letter $S$ is reserved for a set of $s=|S|$ pairwise nonadjacent or independent vertices of $G$. The independence number, $\alpha(G)$, is the maximum of the cardinalities $s$ of the independent sets $S$ in $G$. The italic numbers 0 and 1 denote all-ones column vectors and all-zeros column vectors, respectively. The notation $1_{T}$ denotes the characteristic column vector of a subset $T$ of $V$. Because $S$ is independent, $1_{S}^{\top} W 1_{S}=0$ for every weight matrix $W$ of $G$ and $1_{S}^{\top} W 1_{S} \leq 0$ for every extended weight matrix $W$ of $G$.

*Received by the editors January 23, 2009. Accepted for publication July 31, 2010. Handling Editor: Richard A. Brualdi.

†Department of Mathematics, Royal Military College, PO Box 17000, Station Forces, Kingston, Ontario K7K 7B4, Canada (rjelzinga@gmail.com). Research supported by NSERC Canada and by Queen's McLaughlin and Baumann graduate fellowships.

${ }^{\ddagger}$ Department of Mathematics and Statistics, Queen’s University, Kingston, Ontario K7L 3N6, Canada (gregoryd@mast.queensu.ca). Research supported by NSERC Canada. 
A matrix $M$ is $r$-regular if each of its row sums equals $r$, equivalently, if $M 1=r 1$. Thus, $A(G)$ is $r$-regular if and only if $G$ is $r$-regular, that is, if and only if each vertex of $G$ has degree $r$.

The greatest and least eigenvalues of a symmetric matrix will be denoted by $\lambda_{1}$ and $\lambda_{n}$, respectively. If $W$ is an extended weight matrix, then $\lambda_{1}(W)>0>\lambda_{n}(W)$. For if $\lambda_{1}(W) \leq 0$ or $\lambda_{n}(W) \geq 0$, then the eigenvalues of $W$ would all be 0 since they sum to $0=\operatorname{trace}(W)$. But then $W$ would be a zero matrix, a contradiction.

A ratio bound on $\alpha(G)$ attributed to A. Hoffman (unpublished, see [13, p. 16]) states that if $G$ is $r$-regular and $A$ is the adjacency matrix of $G$, then

$$
\alpha(G) \leq \frac{\left|\lambda_{n}(A)\right|}{r+\left|\lambda_{n}(A)\right|} n .
$$

Here, $r=\lambda_{1}(A)$. The bound (1.1) was proved earlier by P. Delsarte [7, p. 46, 50] for graphs in association schemes.

It is tempting to conjecture that an extension of Hoffman's bound (1.1) that includes nonregular graphs might be $\alpha(G) \leq n\left|\lambda_{n}(A)\right| /\left(\delta(G)+\left|\lambda_{n}(A)\right|\right)$ where $\delta(G)$ is the minimum vertex degree of $G$, but this fails for the complete bipartite graphs $K_{s, t}$ when $s>t$. However, W. H. Haemers [14, p. 15] (see also [15, p. 597]) extended Hoffman's bound (1.1) by showing that

$$
\alpha(G) \leq \frac{\left|\lambda_{1}(A) \lambda_{n}(A)\right|}{\delta(G)^{2}+\left|\lambda_{1}(A) \lambda_{n}(A)\right|} n
$$

This bound not only agrees with (1.1) when $G$ is regular, but also attains equality for every semiregular bipartite graph, that is, for every bipartite graph for which the degrees of the vertices in each part are constant (see Lemma 2.10).

Ratio bounds such as (1.1) and (1.2) can often be modified to allow weight matrices or extended weight matrices. Such generalizations will be called weighted ratio bounds. For example, Haemers observed that (1.1) generalizes to graphs with $r$-regular weight matrices (Remark 2.5). In fact, Godsil and Newman show that (1.1) holds for graphs with $r$-regular extended weight matrices (Corollary 3.3).

Special types of weight matrices or extended weight matrices are needed for some of the bounds that are presented. The graphs that have such matrices are characterized in Lemma 2.6 (regular weight matrices), Remark 2.12 (nonnegative semiregular weight matrices), Lemma 3.4 (regular extended weight matrices) and Remark 5.5 (nonnegative regular weight matrices).

In Sections 2 and 3, three main examples of weighted ratio bounds are presented: Lemmas 2.1, 3.1 and 3.6. Other bounds follow from these three as special cases. Relations to Schrijver's parameter $\vartheta^{\prime}$ appear in Section 4 and relations to Shannon's 
capacity $\Theta$, Lovász' parameter $\vartheta$, and the ultimate independence ratio for categorical products appear in Section 5. The presentation touches on work of Haemers [15], and recent work of Godsil and Newman [10], Newman [23], Luz et al [20, 21, 22] and Alon et al [1].

In Section 6, the paper concludes with some comments on a weighted version of the Cvetković bound (also known as the inertia bound) that appear in the Ph.D. thesis [8] of the first author.

2. Weighted ratio bounds from eigenvalue interlacing. For an $n \times n$ matrix $M$ and subset $T$ of $\{1,2, \ldots, n\}$, let $\bar{r}_{T}(M)$ denote the average of the row sums of $M$ that are indexed by $T$. Thus,

$$
\bar{r}_{T}(M)=\frac{1_{T}^{\top} M 1}{|T|} .
$$

For $0<a \leq b \leq n$, let

$$
\delta_{a}(M)=\min \left\{\left|\bar{r}_{T}(M)\right|:|T|=a\right\} \quad \text { and } \delta_{a, b}(M)=\min \left\{\left|\bar{r}_{T}(M)\right|: a \leq|T| \leq b\right\} .
$$

Lemma 2.1 below provides a weighted generalization of (1.2) that exploits known estimates on the independence number. In the lemma, if $W$ is the adjacency matrix $A$ of $G$ and $a=1$, then $\delta_{a, b}(A)=\delta(G)$, and the bound (2.1) becomes (1.2).

Lemma 2.1. For each weight matrix $W$ of $G$, and each pair $a, b$ of positive integers such that $a \leq \alpha(G) \leq b<n$,

$$
\alpha(G) \leq \frac{\left|\lambda_{1}(W) \lambda_{n}(W)\right|}{\delta_{a, b}(W)^{2}+\left|\lambda_{1}(W) \lambda_{n}(W)\right|} n .
$$

If equality holds, then the rows and columns of $W$ may be reindexed to give a symmetric block matrix of the form

$$
\left[\begin{array}{cc}
O & B \\
B^{\top} & C
\end{array}\right]
$$

where $O$ is a zero matrix of order $\alpha(G), B$ has constant row sums all equal to $\delta_{a, b}(W)$ or all equal to $-\delta_{a, b}(W)$, and $B^{\top}$ and $C$ also have constant row sums.

Proof. The proof follows that of [15, p. 597], but with $W$ in place of $A$. Let $S$ be a maximum independent set of $s=\alpha(G)$ vertices in $G$ and let $\bar{S}=V \backslash S$ be the set of vertices of $G$ not in $S$. Let $Q$ be the $2 \times 2$ quotient matrix of average row sums of the four submatrices $O=W_{S, S}, B=W_{S, \bar{S}}, B^{\top}=W_{\bar{S}, S}, C=W_{\bar{S}, \bar{S}}$ of $W$ determined by the vertex partition $V=S \cup \bar{S}$. Because $G$ is always assumed to have at least one edge, $s<n$. Also, $\bar{r}_{S}=\bar{r}_{S}(W)$ equals the average of the row sums of $B$. Thus,

$$
Q=\left[\begin{array}{cc}
0 & \bar{r}_{S} \\
\frac{s \bar{r}_{S}}{n-s} & *
\end{array}\right] .
$$


Here $\lambda_{1}(Q) \lambda_{2}(Q)=\operatorname{det} Q=-s \bar{r}_{S}^{2} /(n-s) \leq 0$ so $\lambda_{1}(Q) \geq 0 \geq \lambda_{2}(Q)$. By eigenvalue interlacing [15, p. 596], $\lambda_{1}(W) \geq \lambda_{1}(Q) \geq 0 \geq \lambda_{2}(Q) \geq \lambda_{n}(W)$. Therefore,

$$
\frac{s \bar{r}_{S}^{2}}{n-s}=\left|\lambda_{1}(Q) \lambda_{2}(Q)\right| \leq\left|\lambda_{1}(W) \lambda_{n}(W)\right| .
$$

Also, as mentioned earlier, $\left|\lambda_{1}(W) \lambda_{n}(W)\right|>0$. Inequality (2.1) now follows from (2.2) by noting that $\bar{r}_{S}(W)^{2} \geq \delta_{a, b}(W)^{2}$.

Suppose $W$ gives equality in (2.1). Then equality holds in the inequalities above. Thus, $\lambda_{1}(W)=\lambda_{1}(Q)$ and $\lambda_{n}(W)=\lambda_{2}(Q)$. That is, the interlacing is tight [15, p. 594], so each of the submatrices $B, B^{\top}, C$ has constant row sums [15, p. 596]. Also, $\bar{r}_{S}(W)^{2}=\delta_{a, b}(W)^{2}$, so the row sums of $B$ are all equal to $\delta_{a, b}(W)$ or to $-\delta_{a, b}(W)$.

REMARK 2.2. In the expression $\delta_{a, b}(W)=\min \left\{\left|\bar{r}_{T}(W)\right|: a \leq|T| \leq b\right\}$, if one omits sets $T$ that, for one reason or another, are clearly not independent, then a possibly larger value $\delta^{\prime}$ is obtained that satisfies $\bar{r}_{S}(W)^{2} \geq \delta^{\prime 2}$ in (2.2), and $\delta^{\prime}$ may be used in place of $\delta_{a, b}(W)$ in $(2.1)$.

REMARK 2.3. In selecting $b$ in Lemma 2.1, note that each application of (2.1) yields an upper bound on $\alpha$ and so, if $W$ is not regular, may provide an improved value of $b$ with which to apply (2.1) again. In selecting $a$, it may be helpful to keep in mind the following simple lower bound on $\alpha(G)$ due to Caro [3] and Wei [29]. (Of course, small independent sets can also be found by direct inspection.) Construct an independent set $S$ in $G$ by the greedy procedure of successively selecting vertices of minimum degree and deleting their neighbors. It follows by induction and the convexity of the function $f(t)=1 /(1+t)$ that

$$
\alpha(G) \geq|S| \geq \sum_{i=1}^{n} \frac{1}{1+d_{i}} \geq \frac{n}{1+\bar{d}}
$$

where $\bar{d}=\sum_{i} d_{i} / n$, the average of the degrees $d_{1}, \ldots, d_{n}$ of the vertices in $G$. For lower bounds on the independence number of triangle-free graphs, see Shearer [26].

EXAMPLE 2.4. The bound in Lemma 2.1 need not hold for extended weight matrices. For if $G$ is the star $K_{1, n-1}$, then $\alpha=n-1$ and an example of an extended weight matrix $W$ is $I-J$ where $I$ is an identity matrix and $J$ is an all-ones matrix. But $\lambda_{1}(W)=1, \lambda_{n}(W)=1-n$ and $\delta_{a, b}(W)=n-1$ whenever $a \leq \alpha(G) \leq b$, so the upper bound in inequality (2.1) is 1 .

The bound involving regular weight matrices that appears in the following remark is mentioned in the proof of Theorem 3.4 in [15]. A recent result of Godsil and Newman [10, Lem. 2.6] states that the bound in the remark also holds for extended weight matrices (see Corollary 3.3). Bounds that require $r$-regular matrices are useful only if $r>0$, so it is assumed throughout that an $r$-regular weight matrix or extended weight matrix has $r>0$. 
REMARK 2.5. If $W$ is an $r$-regular weight matrix for a graph $G$, then

$$
\alpha(G) \leq \frac{\left|\lambda_{n}(W)\right|}{r+\left|\lambda_{n}(W)\right|} n
$$

For, in the proof of Lemma 2.1, $\bar{r}_{S}=r$ and the unspecified entry $*$ in $Q$ equals $(n-2 s) r /(n-s)$. Thus $Q$ is $r$-regular and $\lambda_{1}(Q)=r$. As before, $\left|\lambda_{2}(Q)\right| \leq\left|\lambda_{n}(W)\right|$ so $s r^{2} /(n-s)=|\operatorname{det} Q|=\left|\lambda_{1}(Q) \lambda_{2}(Q)\right| \leq r\left|\lambda_{n}(W)\right|$ and inequality (2.3) follows.

The next lemma determines the graphs for which the preceding remark is applicable. Because a graph has an $r$-regular weight matrix if and only if each of its connected components does, it is sufficient to consider connected graphs.

Lemma 2.6. A connected graph $G$ has an r-regular weight matrix with $r>0$ if and only if it is not bipartite or if it is bipartite with equal part sizes.

Proof. Let $N=N(G)$ denote the $n \times m$ vertex-edge incidence matrix of a graph $G$ with $n$ vertices and $m$ edges. That is, $N_{i, e}=1$ if vertex $i$ is incident to edge $e$ and $N_{i, e}=0$ otherwise. Weight matrices $W$ of $G$ with prescribed row sum vectors $b=W 1$ correspond to solutions $w$ of $N w=b$ where $w$ is a column $m$-vector whose entries are the weights $W_{i, j}$ on the edges $i j$ of $G$. For connected graphs on $n$ vertices, $\operatorname{rank}(N)=n-1$ if the graph is bipartite and $\operatorname{rank}(N)=n$ otherwise [25, 11]. It follows that if $G$ is connected and not bipartite then the equation $N w=b$ has a solution $w$ for each column $n$-vector $b$, while if $G=G(X, Y)$ is connected and bipartite then $N w=b$ has a solution $w$ if and only if $\sum_{i \in X} b_{i}=\sum_{j \in Y} b_{j}$. Taking $b=r 1$ gives the result stated. $\square$

If $W$ is a weight matrix with nonnegative row sums, then $\bar{r}_{T}(W) \geq 0$ for all subsets $T \subset V$. Thus, if $1 \leq a \leq b<n$, and $r_{\min }(W)$ denotes the minimum row sum of $W$, it follows that

$$
\delta_{a, b}(W)=\delta_{a}(W) \geq \delta_{1}(W)=r_{\min }(W), \quad \text { if } W \text { has nonnegative row sums. }
$$

This implies the following convenient corollary to Lemma 2.1.

Corollary 2.7. For each weight matrix $W$ of $G$ with nonnegative row sums and each positive integer $a \leq \alpha(G)$,

$$
\alpha(G) \leq \frac{\left|\lambda_{1}(W) \lambda_{n}(W)\right|}{\delta_{a}(W)^{2}+\left|\lambda_{1}(W) \lambda_{n}(W)\right|} n \leq \frac{\left|\lambda_{1}(W) \lambda_{n}(W)\right|}{r_{\min }(W)^{2}+\left|\lambda_{1}(W) \lambda_{n}(W)\right|} n .
$$

REMARK 2.8. As in Remark 2.2, omitting from $\delta_{a}(W)=\min \left\{\bar{r}_{T}(W):|T|=a\right\}$ any sets $T$ that, for one reason or another, are clearly not independent, yields a possibly larger value $\delta^{\prime}$ that may be used in place of $\delta_{a}(W)$ in (2.4). 
For matrices $M$ and $N$ of the same size, write $M \geq N$ (respectively, $M>N$ ) if $M_{i, j} \geq N_{i, j}$ (respectively, $M_{i, j}>N_{i, j}$ ) for all row and column indices $i, j$. A matrix $M$ is nonnegative if $M \geq O$, where $O$ denotes a zero matrix.

The next lemma implies that the final bound in (2.4) will not be helpful if nonnegative weight matrices are used for graphs that have small maximum independent sets and vertices with neighbor sets that are small and independent. In particular, if $G$ has a vertex of degree 1, then the final bound in (2.4) is at least $n / 2$ if nonnegative weight matrices are used. (Of course, vertices of degree 1 may be assumed to be in a maximum independent set and so may be deleted).

LEMma 2.9. Let $G$ be a graph on $n$ vertices with a vertex $u$ whose neighbor set is independent. Then the final upper bound in (2.4) is at least $\frac{4 n}{d^{2}+2 d+5}$ where $d$ is the number of neighbors of $u$.

Proof. Let $W$ be a nonnegative weight matrix for $G$. If $r_{\min }(W)=0$, then the final upper bound in (2.4) is $n$. Since $n>4 n /\left(d^{2}+2 d+5\right)$, it may be assumed that $r_{\min }(W)>0$.

If $t$ is the sum of the weights on the edges incident to $u$, then $t$ is a line sum of $W$ so $t \geq r_{\min }(W)>0$. Let $B$ be the $(d+1) \times(d+1)$ principal submatrix of $W$ indexed by $u$ and its neighbors. By the interlacing eigenvalues theorem for bordered matrices [17, p. 554], $\lambda_{n}(W) \leq \lambda_{d+1}(B) \leq \lambda_{1}(B) \leq \lambda_{1}(W)$ where $\lambda_{1}(B)=-\lambda_{d+1}(B)$ since $\operatorname{trace}(B)=0$ and $\operatorname{rank} B=2$. Also, using a Rayleigh-Ritz ratio [17, p. 176], $\lambda_{1}(B) \geq 1^{\top} B 1 / 1^{\top} 1=2 t /(d+1)$. Thus, $\left|\lambda_{1}(W) \lambda_{n}(W)\right| \geq \lambda_{1}(B)^{2} \geq 4 t^{2} /(d+1)^{2} \geq$ $4 r_{\min }(W)^{2} /(d+1)^{2}$ and the stated inequality follows.

A weight matrix $W$ for a bipartite graph $G(X, Y)$ with vertex parts $X, Y$ is $\left(r_{X}, r_{Y}\right)$-semiregular if the row sums of $W$ corresponding to vertices in $X$ all equal $r_{X}$ and the row sums corresponding to vertices in $Y$ all equal $r_{Y}$.

The next lemma provides graphs where equality is attained in Corollary 2.7. As mentioned earlier after (1.2), the lemma implies that every semiregular bipartite graph attains equality in (1.2).

Lemma 2.10. Let $G(X, Y)$ be a bipartite graph. If $G(X, Y)$ has a nonnegative semiregular weight matrix $W$, then $W$ attains equality throughout (2.4).

Proof. For suppose that $G$ has a nonnegative $\left(r_{X}, r_{Y}\right)$-semiregular weight matrix $W$. Then $r_{X}|X|=r_{Y}|Y|$ and $|X|+|Y|=n$. If $\lambda$ is an eigenvalue of $W$, then $\lambda^{2}$ is an eigenvalue of $W^{2}$. Since $W^{2}$ is nonnegative, $\lambda^{2}$ is bounded by the maximum row sum of $W^{2}\left[17\right.$, p. 346]. But $W^{2}$ is $r_{X} r_{Y}$-regular, so $\left|\lambda_{1}(W) \lambda_{n}(W)\right| \leq r_{X} r_{Y}$. We may assume that $|X| \geq|Y|$. Then $\alpha(G) \geq|X|$ and $r_{Y} \geq r_{X}=r_{\min }(W)$. Substitution in 
(2.4) gives

$$
|X| \leq \alpha(G) \leq \frac{\left|\lambda_{1} \lambda_{n}\right|}{r_{\min }^{2}+\left|\lambda_{1} \lambda_{n}\right|} n \leq \frac{r_{Y}}{r_{X}+r_{Y}} n=\frac{r_{Y}|X|}{r_{Y}|Y|+r_{Y}|X|} n=|X| .
$$

Thus, $\alpha(G)=|X|$ and equality is attained in (2.4).

EXAMPLE 2.11. Every path $P_{n}$ has a nonnegative semiregular weight matrix $W$ and so, by Lemma 2.10, attains the bound (2.4). For, if $n$ is even, let $W$ be the adjacency matrix of a perfect matching in $P_{n}$. But if $n$ is odd, weight the edges of $P_{n}$ according to the pattern in Figure 2.1. Then let $W_{i, j}$ be the weight on edge $i j$ and $W_{i, j}=0$ otherwise. (Additional examples of trees with semiregular weight matrices that give positive weights on the edges may be found in [9]).

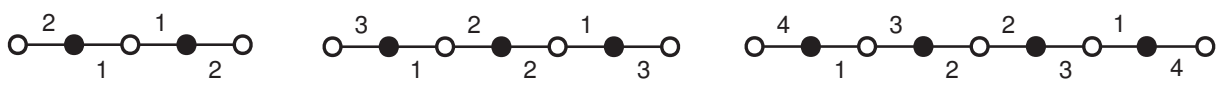

FIG. 2.1. Semiregular edge-weightings of $P_{n}$ for $n=5,7,9$.

REMARK 2.12. It follows from Lemma 2.5 in [9] (with $p_{i}=|Y|, q_{j}=|X|$ for all $i, j)$ that a connected bipartite graph $G(X, Y)$ has a nonnegative semiregular weight matrix if and only if

$$
|X||N(S)| \geq|Y||S| \text { for all subsets } S \subseteq X,
$$

where $N(S)$ denotes the set of all neighbors in $Y$ of vertices in $S$. (As in (3.1) of [9], if the inequality in (2.12) is strict when $\emptyset \varsubsetneqq S \varsubsetneqq X$, then there is a semiregular weight matrix that gives positive weights on the edges of the graph). Thus every bipartite graph that satisfies condition (2.5) has a weight matrix that attains equality in (2.4). Also, if $G$ has a spanning subgraph $H$ with the same independence number as $G$, and $H$ attains the bound in Lemma 2.1 with a weight matrix $W$, then $G$ must also attain the bound with $W$. In particular, every graph $G$ with $\alpha(G)=\lceil n / 2\rceil$ that contains a Hamilton path or a perfect matching attains the bound in Corollary 2.7.

For an $n \times n$ matrix $M$, let $\bar{r}(M)$ denote the average of the row sums of $M$. Thus,

$$
\bar{r}(M)=\bar{r}_{V}(M)=\frac{1^{\top} M 1}{n} .
$$

For $0<a \leq n$, let

$$
\gamma_{a}(M)=\min \left\{\bar{r}_{T}(M):|T|=a\right\} .
$$

Recall that $\delta_{a}(M)=\min \left\{\left|\bar{r}_{T}(M)\right|:|T|=a\right\}$. Thus, $\gamma_{a}(M) \leq \delta_{a}(M)$ with equality when $M \geq O$. Also $\gamma_{1}(M)=r_{\min }(M)$, the minimum row sum of $M$. 
Let $G^{c}$ denote the complement of a graph $G$. Recall that $G$ and $G^{c}$ have the same automorphisms and that the automorphisms may be identified with the permutation matrices $P$ such that $P^{\top} A P=A$ where $A$ is the adjacency matrix of $G$.

Let $\Gamma=\Gamma(G)$ denote both the set of all automorphisms of $G$ and the set of corresponding permutation matrices. In the following lemma, edges and nonedges $i j$ of $G$ are regarded as having weights determined by the corresponding entries $M_{i, j}$ of $M$.

Lemma 2.13. Given an $n \times n$ symmetric matrix $M$ and a graph $G$ with $n$ vertices and automorphism group $\Gamma=\Gamma(G)$, let

$$
\bar{M}=\frac{1}{|\Gamma|} \sum_{P \in \Gamma} P^{\top} M P .
$$

Then, with respect to $\Gamma$, the off-diagonal entries of $\bar{M}$ are constant on edge orbits in $G$ and on edge orbits in $G^{c}$. Also

$$
\lambda_{1}(\bar{M}) \leq \lambda_{1}(M), \quad \lambda_{n}(\bar{M}) \geq \lambda_{n}(M), \bar{r}(\bar{M})=\bar{r}(M), \quad \text { and } \gamma_{a}(\bar{M}) \geq \gamma_{a}(M)
$$

for each positive integer $a \leq n$. In particular, $r_{\min }(\bar{M}) \geq r_{\min }(M)$.

Proof. For $P \in \Gamma, P \Gamma=\Gamma$, so $\bar{M}=P^{\top} \bar{M} P$. Thus, for all standard basis vectors $e_{i}, e_{j}$ and all $P \in \Gamma, e_{i}^{\top} \bar{M} e_{j}=\left(P e_{i}\right)^{\top} \bar{M}\left(P e_{j}\right)$. But, for $i \neq j$, a pair $\left(e_{i}, e_{j}\right)$ corresponds to an edge of $G$ if and only if $\left(P e_{i}, P e_{j}\right)$ does. Thus the entries of $\bar{M}$ corresponding to an edge orbit of $G$ are equal as are entries corresponding to an edge orbit of $G^{c}$.

If $x$ is a $\lambda_{1}$-eigenvector of $\bar{M}$ and $x^{\top} x=1$, then

$$
\lambda_{1}(\bar{M})=x^{\top} \bar{M} x=\frac{1}{|\Gamma|} \sum_{P \in \Gamma}(P x)^{\top} M(P x) \leq \lambda_{1}(M)
$$

where the final inequality follows because $z^{\top} M z \leq \lambda_{1}(M)$ when $z^{\top} z=1$ [17, p. 180]. Applying the inequality to $-\bar{M}$ gives $\lambda_{n}(\bar{M}) \geq \lambda_{n}(M)$.

Because $1^{\top} M 1=1^{\top} P^{\top} M P 1$, the equality $\bar{r}(\bar{M})=\bar{r}(M)$ follows from (2.6). If $1_{T}$ is the characteristic function of a subset of $V$ of size $a$, then $P 1_{T}$ is as well. Thus,

$$
\bar{r}_{T}(\bar{M})=\frac{1}{a} 1_{T}^{\top} \bar{M} 1_{T}=\frac{1}{|\Gamma|} \sum_{P \in \Gamma} \frac{1}{a}\left(P 1_{T}\right)^{\top} M\left(P 1_{T}\right) \geq \gamma_{a}(M) .
$$

Therefore, $\gamma_{a}(\bar{M}) \geq \gamma_{a}(M)$.

The following corollary implies that the entries of a nonnegative weight matrix $W$ in Corollary 2.7 may be assumed to be constant on edge orbits of the automorphism group $\Gamma$ of the graph $G$. In particular, if $\Gamma$ is transitive on the edges of $G$, 
then the corollary implies that a nonnegative weight matrix in Corollary 2.7 gives no improvement over the adjacency matrix.

Corollary 2.14. Let $W$ be a nonnegative weight matrix for a graph $G$, and let $\bar{W}$ be the weight matrix obtained from $W$ in (2.6). Then, whenever $0<a \leq \alpha(G)$,

$$
\alpha(G) \leq \frac{\left|\lambda_{1}(\bar{W}) \lambda_{n}(\bar{W})\right|}{\delta_{a}(\bar{W})^{2}+\left|\lambda_{1}(\bar{W}) \lambda_{n}(\bar{W})\right|} n \leq \frac{\left|\lambda_{1}(W) \lambda_{n}(W)\right|}{\delta_{a}(W)^{2}+\left|\lambda_{1}(W) \lambda_{n}(W)\right|} n
$$

In particular, the inequalities hold for $\delta_{1}(W)=r_{\min }(W)$.

Proof. The first inequality in (2.7) is proved in Corollary 2.7.

Because $\lambda_{1}(W)>0>\lambda_{n}(W)$ for weight matrices, the inequalities in Lemma 2.13 imply that $\left|\lambda_{1}(\bar{W}) \lambda_{n}(\bar{W})\right| \leq\left|\lambda_{1}(W) \lambda_{n}(W)\right|$. Also, $W \geq O$, so $\delta_{a}(\bar{W})^{2}=\gamma_{a}(\bar{W})^{2} \geq$ $\gamma_{a}(W)^{2}=\delta_{a}(W)^{2}$, and the second inequality in (2.7) follows.

3. Weighted ratio bounds from positive semidefinite matrices. The two examples of weighted ratio bounds considered in this section are obtained using a technique of C. D. Godsil and M. W. Newman [10] (see also [23]). It turns out that extensions of the ratio bound (1.1) can be obtained using the fact that if $W$ is a weight matrix or extended weight matrix of a graph $G$ and $D$ is a diagonal matrix such that $B=W+D$ is positive semidefinite, then $y^{\top} B y \geq 0$ for all $y$, with equality if and only if $B y=0$. The calculations are particularly amenable in the following special case.

For an $n$-vector $x$ and a maximum independent set $S$ of $s=\alpha(G)$ vertices in a graph $G$ with $n$ vertices, let $y=z-s x / x^{\top} x$ where $z_{i}=x_{i}$ for $i \in S$ and $z_{i}=0$ otherwise. Let $W$ be a weight matrix or extended weight matrix for $G$ and let $\lambda_{n}=$ $\lambda_{n}(W)$ be the least eigenvalue of $W$. Then $B=W-\lambda_{n} I$ is positive semidefinite. If $W$ is a weight matrix, then $z^{\top} W z=0$. If $W$ is an extended weight matrix and $z \geq 0$, then $z^{\top} W z \leq 0$. Thus, if $W$ is a weight matrix and $x$ is arbitrary or if $W$ is an extended weight matrix and $x_{i} \geq 0$ for $i \in S$, then

$$
\begin{aligned}
0 & \leq y^{\top} B y=y^{\top} W y-\lambda_{n} y^{\top} y \\
& \leq-2 s \frac{x^{\top} W z}{x^{\top} x}+s^{2} \frac{x^{\top} W x}{\left(x^{\top} x\right)^{2}}-\lambda_{n}\left(z^{\top} z-2 s \frac{x^{\top} z}{x^{\top} x}+\frac{s^{2}}{x^{\top} x}\right)
\end{aligned}
$$

with equality in the second line when $W$ is a weight matrix.

Choosing $x$ in inequality (3.1) to be an eigenvector of $W$ gives another generalization of Hoffman's regular ratio bound (1.1). This is stated in the following lemma. There, $\sum_{i \in S} x_{i}^{2}<x^{\top} x$ and the bound is useful only if $\lambda_{k}(W)>0$.

Lemma 3.1. Let $W$ be a weight matrix of $G$ that has an eigenvector $x$ that has a nonzero entry on some maximum independent set $S$ of $s$ vertices. If $\lambda_{k}$ is the 
eigenvalue associated with $x$, and $x$ is scaled so that $\sum_{i \in S} x_{i}^{2} \geq s$, then

$$
\alpha(G) \leq \frac{\left|\lambda_{n}(W)\right|}{\lambda_{k}(W)+\left|\lambda_{n}(W)\right|} x^{\top} x
$$

The inequality also holds when $W$ is an extended weight matrix if $x$ satisfies the additional condition that $x_{i} \geq 0$ for $i \in S$.

Proof. It is sufficient to prove the inequality when $\sum_{i \in S} x_{i}^{2}=s$. Then $x^{\top} z=$ $z^{\top} z=s$ and, in (3.1), $x^{\top} W x=\lambda_{k} x^{\top} x$ and $x^{\top} W z=\lambda_{k} x^{\top} z=s \lambda_{k}$, so

$$
0 \leq-\frac{2 s^{2} \lambda_{k}}{x^{\top} x}+\frac{s^{2} \lambda_{k}}{x^{\top} x}-\lambda_{n} s+\frac{2 s^{2} \lambda_{n}}{x^{\top} x}-\frac{s^{2} \lambda_{n}}{x^{\top} x}=-\frac{s^{2} \lambda_{k}}{x^{\top} x}-\lambda_{n} s+\frac{s^{2} \lambda_{n}}{x^{\top} x},
$$

and the stated inequality follows.

REMARK 3.2. If $W$ is a nonnegative weight matrix of $G$ and the subgraph of $G$ induced by the edges $i j$ with $W_{i, j}>0$ is spanning and connected, then $W$ has a $\lambda_{1}$-eigenvector $x \geq 1$ and the bound (3.2) holds with $\lambda_{k}=\lambda_{1}$.

If $W$ is an $r$-regular weight matrix or extended weight matrix for $G$, then $W 1=$ $r 1$ and the bound (3.2) holds with $x=1$ and $\lambda_{k}=r$. This choice implies, in the following corollary, that the bound in Remark 2.5 extends to $r$-regular extended weight matrices. The corollary first appears in an equivalent form involving positive semidefinite matrices as Theorem 6.1 in Godsil and Newman [10] with $B=W-$ $\lambda_{n}(W) I$. It was also observed by Luz [20] for extended weight matrices for which $x=1$ is a $\lambda_{1}$-eigenvector. The corollary also follows from Corollary 4.2 in Section 4 .

COROLlaRY 3.3. If $W$ is an r-regular extended weight matrix for $G$, then

$$
\alpha(G) \leq \frac{\left|\lambda_{n}(W)\right|}{r+\left|\lambda_{n}(W)\right|} n
$$

Every graph has an $r$-regular extended weight matrix with $r<0$ : take $W_{i j}=-1$ for all $i \neq j$. However, as mentioned earlier, bounds such as (3.3) are useful only if $r>0$. The next lemma determines the graphs for which (3.3) is applicable. As in Lemma 2.6, it is sufficient to consider connected graphs.

LEMMA 3.4. Let $G$ be a connected graph and let $r>0$. Then $G$ has an $r$-regular extended weight matrix if and only if $G$ is not a star $K_{1, n-1}$ with $n \geq 3$.

Proof. It is straightforward to check that if $n \geq 3$, the star $K_{1, n-1}$ has no $r$-regular extended weight matrix with $r>0$, so it remains to show that all other connected graphs do have one.

If the connected graph $G$ is not bipartite or if it is bipartite with equal part sizes, then $G$ has an $r$-regular weight matrix by Lemma 2.6, and this is also an $r$-regular 
extended weight matrix. This leaves the case where $G=G(X, Y)$ is connected and bipartite with $|Y|>|X| \geq 2$, say.

Let $b$ be a column $n$-vector with $b_{i}=|Y|$ if $i \in X$ and $b_{j}=|X|$ if $j \in Y$. Then $\sum_{i \in X} b_{i}=|X||Y|=\sum_{j \in Y} b_{j}$. By the observations in the proof of Lemma 2.6, the equation $N w=b$ has a solution and so $G$ has a $(|Y|,|X|)$-semiregular weight matrix; that is, the sum of the weights on edges incident to a vertex in $X$ is always $|Y|$, while the sum of the weights on edges incident to a vertex in $Y$ always equals $|X|$. Give each nonedge with both ends in $X$ a negative weight of $(|X|-|Y|) /(|X|-1)$ and every other nonedge in $G$ a weight of zero. Then the sum of the weights on edges and nonedges incident to each vertex in $G$ is equal to $|X|$. Multiply all the weights by $r /|X|$. Then the associated $n \times n$ matrix $W$ is an $r$-regular extended weight matrix.

REMARK 3.5. The inequalities in Lemma 2.13 imply that if $W$ is an $r$-regular extended weight matrix of $G$, then $\bar{W}$ is as well and $0>\lambda_{n}(\bar{W}) \geq \lambda_{n}(W)$. Thus no loss occurs in the bound (3.3) if $W$ is replaced by $\bar{W}$. Therefore, in Corollary 3.3, W may be assumed to be constant on edge orbits in $G$ and in $G^{c}$.

A second application of (3.1) yields a simple weighted variant of a ratio bound proved by Godsil and Newman [10, Cor. 3.2] for adjacency matrices. As before, let $\bar{r}_{T}(W)=1_{T}^{\top} W 1 /|T|$, the average of the row sums of $W$ indexed by $T$, let $\bar{r}(W)=$ $1^{\top} W 1 / n$, the average row sum of $W$ and, for $0<a \leq \alpha(G)$, let $\gamma_{a}(W)=\min \left\{\bar{r}_{T}(W)\right.$ : $|T|=a\}$. Of course, the bounds in Lemma 3.6 are only of interest when $2 \gamma_{a}(W)>$ $\bar{r}(W)$.

Lemma 3.6. Let $W$ be an extended weight matrix of $G$ and let $S$ be a maximum independent set of vertices. If a is a positive integer such that $a\left\langle\alpha(G)\right.$ and $2 \gamma_{a}(W)>$ $\bar{r}(W)$, then

$$
\begin{aligned}
\alpha(G) & \leq \frac{\left|\lambda_{n}(W)\right|}{2 \bar{r}_{S}(W)-\bar{r}(W)+\left|\lambda_{n}(W)\right|} n \\
& \leq \frac{\left|\lambda_{n}(W)\right|}{2 \gamma_{a}(W)-\bar{r}(W)+\left|\lambda_{n}(W)\right|} n
\end{aligned}
$$

Proof. Let $s=|S|=\alpha(G)$ and $x=1$. Then in (3.1), $z=1_{S}, z^{\top} z=x^{\top} z=s$, $x^{\top} x=n, x^{\top} W x=n \bar{r}$ and $z^{\top} W x=s \bar{r}_{S}$, so

$$
0 \leq-2 \frac{s^{2} \bar{r}_{S}}{n}+\frac{s^{2} \bar{r}}{n}-\lambda_{n}\left(s-\frac{2 s^{2}}{n}+\frac{s^{2}}{n}\right)
$$

and inequality (3.4) follows. Because $\gamma_{a}(W) \leq \bar{r}_{S}(W)$, inequality (3.5) also holds.

REMARK 3.7. By Lemma 2.13, the ratio $\left(2 \gamma_{a}(W)-\bar{r}(W)\right) /\left|\lambda_{n}(W)\right|$ will not decrease when $W$ is replaced by $\bar{W}$. Thus, for the final upper bound on $\alpha(G)$ in (3.5), 
it may be assumed that the entries of $W$ are constant on edge orbits and nonedge orbits of the automorphism group $\Gamma$.

REMARK 3.8. In Lemma 3.6, if $W$ is an $r$-regular extended weight matrix, then $2 \gamma_{a}(W)-\bar{r}(W)=r$ and Corollary 3.3 is obtained once again. Also, as in Remark 2.2 , omitting from the expression $\gamma_{a}(W)=\min \left\{\bar{r}_{T}(W):|T|=a\right\}$ any sets $T$ that are clearly not independent, yields a possibly larger value $\gamma^{\prime} \leq \bar{r}_{S}(W)$ that may be used in place of $\gamma_{a}(W)$ in (3.5).

Throughout this paper, weight and extended weight matrices are always assumed to have zero diagonal. It should be mentioned that some work has been done using matrices with nonnegative diagonal entries. Let $G$ be a simple graph and suppose that an $r$-regular graph is formed from $G$ by attaching $l$ loops. If $A$ is the adjacency matrix of the resulting graph (so $A_{i, i}$ is the number of loops at vertex $i, A 1=r 1$ and $\operatorname{trace}(A)=l$ ), Godsil and Newman [10] have shown that

$$
\alpha(G) \leq \frac{-\lambda_{n}(A)+\sqrt{\lambda_{n}(A)^{2}+\frac{4 l}{n}\left(r-\lambda_{n}(A)\right)}}{\frac{2}{n}\left(r-\lambda_{n}(A)\right)} .
$$

The bound is proved by taking $B=A-\lambda_{n}(A) I, x=1$ and $z=1_{S}$ in (3.1), noting that $z^{\top} A z \leq l$, and by examining a quadratic inequality.

In [6] it is shown that the bound in (3.6) is also an upper bound on $\vartheta(G)$ (defined in Section 5). The bound has yielded good upper bounds on $\alpha(G)$ for some classes of simple graphs that may be made regular by attaching a small number of loops $[6,10]$.

REMark 3.9. The bound (3.6) has a weighted analogue. Let $W$ be an extended weight matrix of a simple graph $G$. Choose a nonnegative diagonal matrix $D$ so that $W+D$ is $r$-regular for some $r>0$. Noting that $1_{S}^{\top} W 1_{S} \leq 0$, it is straightforward to check that the bound (3.6) continues to hold if $A$ is replaced by $W+D$ and $l$ by $\operatorname{trace}(D)$.

4. Schrijver's parameter $\vartheta^{\prime}(G)$. In [27], A. Schrijver shows that

$$
\alpha(G) \leq \vartheta^{\prime}(G) \text { where } \vartheta^{\prime}(G)=\min _{K, W} \lambda_{1}(K-W)=\min _{W} \lambda_{1}(J-W)
$$

and the minima are taken over all extended weight matrices $W$ of $G$ and, in the first minimum, also over all matrices $K \geq J$, the all-ones matrix. The last equality holds because the greatest eigenvalue of a matrix is not increased by decreasing its diagonal entries. By Lemma 2.13, the extended weight matrices $W$ in the second minimum of (4.1) may be restricted to those whose entries are constant on edge orbits and nonedge orbits of $G$. 
Luz [20, p. 105] has shown that, for all extended weight matrices $W$ of $G$,

$$
\begin{gathered}
\vartheta^{\prime}(G) \leq \nu(G, W) \text { where } \\
\nu(G, W)=\max \left\{21^{\top} x-x^{\top}(H+I) x: x \in \mathbb{R}^{n}\right\}
\end{gathered}
$$

and where $H+I$ is the positive semidefinite matrix $\frac{W}{\left|\lambda_{n}(W)\right|}+I$. Note that for all extended weight matrices $W$ of $G$ and all maximum independent subsets $S$, the inequality $\nu(G, W) \geq \alpha(G)-\sum_{i, j \in S} W_{i, j} \geq \alpha(G)$ follows by taking $x=1_{S}$ in (4.3).

In Lemma 4.1 below, it is seen that for many weight matrices or extended weight matrices $W$, the maximum problem (4.3) is the dual of a corresponding minimum problem. In (4.5), this yields upper bounds on $\vartheta^{\prime}(G)$ and so, by (4.1), on $\alpha(G)$. The lemma is proved in $[21$, p. 310] for the special case where $W$ is an adjacency matrix.

LEMma 4.1. For an extended weight matrix $W$ of $G$, let $\lambda_{1}(W) \geq \lambda_{2}(W) \geq$ $\cdots \geq \lambda_{m}(W)$ be the eigenvalues of $W$ that are strictly greater than $\lambda_{n}(W)$, and let $\mathcal{U}$ be the span of a set $u_{1}, u_{2}, \ldots, u_{m}$ of corresponding orthonormal eigenvectors. If $\left(W-\lambda_{n}(W) I\right) y>0$ for some column n-vector y (for example, if $W$ is a nonnegative weight matrix) then

$$
\nu(G, W)=\min \left\{\sum_{i=1}^{m} \frac{\left|\lambda_{n}(W)\right|}{\lambda_{i}(W)+\left|\lambda_{n}(W)\right|}\left(x^{\top} u_{i}\right)^{2}: x \geq 1, x \in \mathcal{U}\right\}
$$

Proof. Let $Q=H+I=\frac{W}{\left|\lambda_{n}(W)\right|}+I$ and $a=1$ in Lemma 7.1 in the Appendix. Then $Q$ is positive semidefinite and $Q x=\frac{1}{\left|\lambda_{n}(W)\right|}\left(W-\lambda_{n}(W)\right) x>1$ for some scalar multiple $x$ of $y$, so $\nu(G, W)=\min \left\{z^{\top} D^{-1} z: z \in \mathbb{R}^{m}, U z \geq 1\right\}$ by (4.3) and Lemma 7.1. The positive eigenvalues of $Q$ are $\lambda_{i}(Q)=\frac{\lambda_{i}(W)}{\left|\lambda_{n}(W)\right|}+1$ for $i=1, \ldots, m$. The eigenvectors $u_{1}, u_{2}, \ldots, u_{m}$ of $W$ are also eigenvectors of $Q$.

The vectors $U z$ with $U z \geq 1$ for some $z \in \mathbb{R}^{m}$ are precisely the vectors $x \in \mathcal{U}$ with $x \geq 1$. Setting $x=U z$ gives $U^{\top} x=U^{\top} U z=I z=z$, so

$z^{\top} D^{-1} z=x^{\top} U D^{-1} U^{\top} x=x^{\top}\left(\sum_{i=1}^{m} \lambda_{i}(Q)^{-1} u_{i} u_{i}^{\top}\right) x=\sum_{i=1}^{m} \frac{\left|\lambda_{n}(W)\right|}{\lambda_{i}(W)+\left|\lambda_{n}(W)\right|}\left(x^{\top} u_{i}\right)^{2}$

Equality (4.4) now follows from (4.3) and Lemma 7.1.

In the statement of Lemma 4.1, because $x$ is in $\mathcal{U}$ (the span of the orthonormal eigenvectors $u_{1}, \ldots, u_{m}$ of $\left.W\right)$, it follows that $x=\sum_{i=1}^{m}\left(x^{\top} u_{i}\right) u_{i}$. Let $a_{i}(x)=$ $\left(x^{\top} u_{i}\right)^{2} / x^{\top} x, i=1, \ldots, m$. Then the $a_{i}(x)$ are nonnegative numbers that sum to 1 . Thus, whenever $W$ is an extended weight matrix for $G$ that has a vector $x \geq 1$ in $\mathcal{U}$ 
(equivalently, if $\left(W-\lambda_{n}(W) I\right) y>0$ for some $\left.y \in \mathbb{R}^{n}\right)$ then, by (4.1), (4.2) and (4.4), $\alpha(G)$ is bounded above by a convex combination of $m=\operatorname{rank}\left(W-\lambda_{n}(W) I\right)$ ratios:

$$
\alpha(G) \leq \vartheta^{\prime}(G) \leq \nu(G, W) \leq \sum_{i=1}^{m} a_{i}(x) \frac{\left|\lambda_{n}(W)\right|}{\lambda_{i}(W)+\left|\lambda_{n}(W)\right|} x^{\top} x .
$$

If $W$ has an eigenvector $x \geq 1$, then (4.5) yields the following corollary. As a bound on $\alpha(G)$, the corollary is a special case of Lemma 3.1. For the bound on $\vartheta^{\prime}$, a self-contained proof that bypasses the previous duality argument is included. The proof is a slight modification of that of Theorem 9 in [18].

COROLlaRY 4.2. If $W$ is an extended weight matrix of $G$ that has an eigenvector $x \geq 1$ with eigenvalue $\lambda_{k}(W)>\lambda_{n}(W)$, then

$$
\alpha(G) \leq \vartheta^{\prime}(G) \leq \frac{\left|\lambda_{n}(W)\right|}{\lambda_{k}(W)+\left|\lambda_{n}(W)\right|} x^{\top} x
$$

Proof. Let $\lambda_{1} \geq \cdots \geq \lambda_{n}$ be the eigenvalues of $W$. Because $x x^{\top} \geq 11^{\top}=J$, the choice $K=x x^{\top}$ may be made in (4.1). Then, for all $t>0$,

$$
\alpha(G) \leq \vartheta^{\prime}(G) \leq \lambda_{1}\left(x x^{\top}-t W\right) .
$$

Note that $x$ is an eigenvector of $K-t W$ with eigenvalue $x^{\top} x-t \lambda_{k}$. Any remaining eigenvector $z$ in an eigenvector basis for $W$ may be chosen to be orthogonal to $x$ and so will also be an eigenvector for $K-t W$ because $K z=0$. Thus, the remaining eigenvalues of $K-t W$ are $-t \lambda_{i}, i \neq k$. For $t=x^{\top} x /\left(\lambda_{k}-\lambda_{n}\right)$, the $n$ eigenvalues of $K-t W$ are

$$
\frac{-\lambda_{n}}{\lambda_{k}-\lambda_{n}} x^{\top} x \text { and } \frac{-\lambda_{i}}{\lambda_{k}-\lambda_{n}} x^{\top} x \text { for } i \neq k .
$$

The largest of these is obtained when $i=n$. The bound on $\vartheta^{\prime}$ in (4.6) now follows from (4.7).

In Corollary 4.2, it is clear that if $\vartheta^{\prime}(G)$ equals the upper bound in (4.6), then at least one entry of $x$ equals 1 . The next example shows that it is not necessary that $x=1$ for equality to hold throughout (4.6).

EXAMPLE 4.3. If a bipartite graph $G$ has a nonnegative semiregular weight matrix $W$ (in particular, if $G$ is edge-transitive), then $W$ has a $\lambda_{1}(W)$-eigenvector $x$ for which $\alpha(G)=\vartheta^{\prime}(G)=\frac{\left|\lambda_{n}(W)\right|}{\lambda_{1}(W)+\left|\lambda_{n}(W)\right|} x^{\top} x$.

For suppose that $W$ is a nonnegative $\left(r_{X}, r_{Y}\right)$-semiregular weight matrix for $G=$ $G(X, Y)$ and that $|X| \geq|Y|$, say. Then $\alpha(G) \geq|X|$ and $r_{X}|X|=r_{Y}|Y|$, so $r_{X} \leq r_{Y}$. 
As observed earlier in Lemma 2.10, $|\lambda| \leq \sqrt{r_{X} r_{Y}}$ for each eigenvalue $\lambda$ of $W$. Let $x$ be the vector with $x_{i}=1$ for $i \in X$ and $x_{j}=\sqrt{r_{Y} / r_{X}}$ for $j \in Y$. Then $x \geq 1$ and it is straightforward to check that $x$ is an eigenvector of $W$ with eigenvalue $\lambda_{1}=\sqrt{r_{X} r_{Y}}$. By (4.6),

$$
\alpha(G) \leq \frac{\left|\lambda_{n}\right|}{\lambda_{1}+\left|\lambda_{n}\right|} x^{\top} x \leq \frac{\sqrt{r_{X} r_{Y}}}{2 \sqrt{r_{X} r_{Y}}}\left(|X|+\frac{r_{Y}}{r_{X}}|Y|\right)=\frac{1}{2}(|X|+|X|)=|X| .
$$

Thus, $\alpha(G)=|X|$ and equality holds in (4.6).

In Section 5, Lovász' parameter $\vartheta(G)$ is defined and observed to be greater than or equal to $\vartheta^{\prime}(G)$. It will be seen there (in Remark 5.2), that the equality in the following lemma refines a corresponding well-known equality of Lovász for $\vartheta(G)$.

LEMMA 4.4. Let $G$ be an edge-transitive graph with adjacency matrix $A$. If $G$ is also $r$-regular for some $r$ (for example, if $G$ also contains an odd cycle), then

$$
\vartheta^{\prime}(G)=\frac{\left|\lambda_{n}(A)\right|}{r+\left|\lambda_{n}(A)\right|} n
$$

Proof. By Lemma 2.13, $\lambda_{1}(\overline{J-W}) \leq \lambda_{1}(J-W)$ for all extended weight matrices $W$ of $G$. Also, the entries of $\overline{J-W}=J-\bar{W}$ are constant on edge orbits and nonedge orbits of $G$. Because $G$ is edge-transitive, this implies that $J-\bar{W}=J-(a A-b(J-$ $I-A))=(b+1) J-(a+b) A-b I$ for some constants $a, b$ with $b \geq 0$. Thus, by (4.1),

$$
\vartheta^{\prime}(G)=\min \left\{\lambda_{1}((b+1) J-(a+b) A-b I): a, b \in \mathbb{R}, b \geq 0\right\} .
$$

Because $G$ is edge-transitive it has at most two vertex degrees, and both occur on each edge of $G$. Thus, if $G$ contains an odd cycle, $G$ must be $r$-regular for some $r$. Then $\mu=(b+1) n-(a+b) r-b$ is an eigenvalue of $J-\bar{W}$ with eigenvector 1 . The remaining vectors in an eigenvector basis may be chosen to be orthogonal to 1 and so have corresponding eigenvalues $-(a+b) \lambda_{i}(A)-b, i=2, \ldots n$.

If $\mu=(b+1) n-(a+b) r-b$ is the greatest eigenvalue of $J-\bar{W}$, then $\mu \geq$ $-(a+b) \lambda_{n}(A)-b$, so $a+b \leq \frac{b+1}{r+\left|\lambda_{n}(A)\right|} n$. In that case, $\lambda_{1}(J-\bar{W})=\mu \geq \frac{(b+1)\left|\lambda_{n}(A)\right|}{r+\left|\lambda_{n}(A)\right|} n \geq$ $\frac{\left|\lambda_{n}(A)\right|}{r+\left|\lambda_{n}(A)\right|} n$ and equality may be attained by taking $b=0$ and $a=n /\left(r+\left|\lambda_{n}(A)\right|\right)$.

If some eigenvalue $-(a+b) \lambda_{i}(A)-b, i=2, \ldots n$ is greatest, then $\mu \leq-(a+$ b) $\lambda_{i}(A)-b$, so $(b+1) n \leq(a+b)\left(r-\lambda_{i}(A)\right)$ where $a+b>0$ and $r-\lambda_{i}(A)>0$ since $(b+1) n>0$. Thus, the greatest eigenvalue is $(a+b)\left|\lambda_{n}(A)\right|-b$ where $a+b \geq \frac{(b+1) n}{r+\left|\lambda_{n}(A)\right|}$. In this case, $\lambda_{1}(J-\bar{W})=(a+b)\left|\lambda_{n}(A)\right|-b \geq \frac{(b+1)\left|\lambda_{n}(A)\right|}{r+\left|\lambda_{n}(A)\right|} n \geq \frac{\lambda_{n}(A) \mid}{r+\left|\lambda_{n}(A)\right|} n$ and equality may be attained by taking $b=0$ and $a=n /\left(r+\left|\lambda_{n}(A)\right|\right)$. 
5. Iterated graph products. The strong product $G \otimes H$ of graphs $G$ and $H$ is the graph with vertex set $V(G) \times V(H)$ where distinct vertices $(i, j),\left(i^{\prime}, j^{\prime}\right)$ are adjacent if and only if $i$ is adjacent or equal to $i^{\prime}$ in $G$ and $j$ is adjacent or equal to $j^{\prime}$ in $H$.

If $S$ is independent in $G$ and $T$ is independent in $H$, then $S \times T$ is independent in $G \otimes H$. Thus, $\alpha(G \otimes H) \geq \alpha(G) \alpha(H)$.

Let $G^{\bigotimes k}$ denote the $k$-fold strong product of $G$. From the above, it follows that $\sqrt[k]{\alpha\left(G^{\bigotimes k}\right)}$ is monotone increasing. Shannon [28] introduced the parameter

$$
\Theta(G)=\sup _{k} \sqrt[k]{\alpha\left(G^{\bigotimes k}\right)}
$$

and showed that $\Theta(G) \leq \alpha^{*}(G)$, the fractional independence number [18] of $G$. To better estimate $\Theta(G)$, Lovász introduced the finer upper bound

$$
\vartheta(G)=\min _{W} \lambda_{1}(J-W),
$$

where $J$ is the all-ones matrix and the minimum is taken over all weight matrices $W$ of $G$. Lovász [18] showed that

$$
\alpha(G) \leq \Theta(G) \leq \vartheta(G) \leq \alpha^{*}(G)
$$

Clearly, $\vartheta^{\prime}(G) \leq \vartheta(G)$. There may be no consistent inequality between $\vartheta^{\prime}(G)$ and $\Theta(G)$, but we have no examples to confirm this.

Luz and Schrijver [22] have shown that

$$
\vartheta(G)=\min _{W} \nu(G, W)
$$

where $\nu(G, W)$ is defined in (4.3) and the minimum is taken over all weight matrices $W$ of $G$. Thus, the upper bound in (4.5) is also an upper bound for $\vartheta(G)$ if $W$ is restricted to be a weight matrix such that $\left(W-\lambda_{n}(W) I\right) y>0$ for some $y$ (for example, if $W$ is a nonnegative weight matrix). This implies the following lemma. (If the weight matrix $W$ is regular, the lemma can be proved directly using the technique of Corollary 4.2 with $x=1$ and $x x^{\top}=11^{\top}=J$.)

Lemma 5.1. If $W$ is a weight matrix of $G$ that has an eigenvector $x \geq 1$ with eigenvalue $\lambda_{k}>\lambda_{n}$, then

$$
\alpha(G) \leq \Theta(G) \leq \vartheta(G) \leq \frac{\left|\lambda_{n}(W)\right|}{\lambda_{k}(W)+\left|\lambda_{n}(W)\right|} x^{\top} x
$$

Thus, if $\alpha(G)$ attains the final upper bound in (5.1) for some weight matrix $W$ and eigenvector $x \geq 1$, then $\Theta(G)=\alpha(G)$. 
REMARK 5.2. If $G$ is an edge-transitive graph with adjacency matrix $A$ and $G$ is $r$-regular, then

$$
\vartheta(G)=\frac{\left|\lambda_{n}(A)\right|}{r+\left|\lambda_{n}(A)\right|} n .
$$

This equality was observed in [18, p. 5]. Because $\vartheta^{\prime}(G) \leq \vartheta(G)$, it follows from Lemma 5.1 and the stronger result in Lemma 4.4.

REMARK 5.3. If $G$ has an $r$-regular weight matrix $W$, then the inequality

$$
\Theta(G) \leq \frac{\left|\lambda_{n}(W)\right|}{r(W)+\left|\lambda_{n}(W)\right|} n
$$

can be shown to follow directly from Corollary 3.3 without using Lovász' bound $\Theta(G) \leq \vartheta(G)$. Following [15, Thm. 3.4], first note that $W_{k}=\otimes_{k}(t W+I)-\otimes_{k} I$ is a weight matrix for $G^{\bigotimes k}$ with constant row sums $(t r+1)^{k}-1$. Choose $t=$ $1 /\left|\lambda_{n}(W)\right|$. Then $\lambda_{n}\left(W_{k}\right)=-1$ and applying Corollary 3.3 to $G^{\bigotimes k}$ gives the upper bound $\sqrt[k]{\alpha\left(G^{\bigotimes k}\right)} \leq n\left|\lambda_{n}(W)\right| /\left(r+\left|\lambda_{n}(W)\right|\right)$ for all $k$. Thus, $\Theta(G)$ also has this upper bound.

A parameter analogous to $\Theta(G)$ is obtained by taking categorical products. The categorical (or weak) product $G \times H$ of graphs $G$ and $H$ is the graph with vertex set $V(G) \times V(H)$ where distinct vertices $(i, j),\left(i^{\prime}, j^{\prime}\right)$ are adjacent if and only if $i$ is adjacent to $i^{\prime}$ in $G$ and $j$ is adjacent to $j^{\prime}$ in $H$.

If $S$ is independent in $G$ and $T$ is independent in $H$, then $S \times V(H)$ and $V(G) \times T$ are independent in $G \times H$. Thus, $\alpha(G \times H) \geq \max \{\alpha(G)|V(H)|,|V(G)| \alpha(H)\}$.

Let $G^{\times k}$ denote the $k$-fold categorical product of $G$. From the above, it follows that the independence ratio, $i\left(G^{\times k}\right)=\alpha\left(G^{\times k}\right) / n^{k}$, is monotone increasing. The limit, $I_{\times}(G)=\sup _{k} i\left(G^{\times k}\right)$, is called the ultimate categorical independence ratio. This was introduced by Brown, Nowakowski and Rall in [2], and is denoted there by $A(G)$.

The following bound was observed in [1, p. 915] in the special case where $W=A$, the adjacency matrix of a regular graph.

LEMmA 5.4. If a graph $G$ has a nonnegative regular weight matrix $W$, then

$$
\frac{\alpha(G)}{n} \leq I_{\times}(G) \leq \frac{\left|\lambda_{n}(W)\right|}{r(W)+\left|\lambda_{n}(W)\right|}
$$

Thus, equality holds if $\alpha(G)=\frac{\left|\lambda_{n}(W)\right|}{r(W)+\left|\lambda_{n}(W)\right|} n$.

Proof. If $W$ is nonnegative and $r$-regular, then so is each irreducible direct summand of $W$. It follows that $r=\lambda_{1}(W)$, the largest eigenvalue of $W$ ([17, p. 503-508]). 
The $k$-fold tensor product, $\otimes_{k} W$, is an $r^{k}$-regular weight matrix for $G^{\times k}$ with greatest eigenvalue $r^{k}$ and least eigenvalue $\lambda_{n}(W) r^{k-1}$. Thus, by Corollary 3.3 or Remark 2.5,

$$
\frac{\alpha(G)}{n} \leq \frac{\alpha\left(G^{\times k}\right)}{n^{k}} \leq \frac{\left|\lambda_{n}(W) r^{k-1}\right|}{r^{k}+\left|\lambda_{n}(W) r^{k-1}\right|}=\frac{\left|\lambda_{n}(W)\right|}{r+\left|\lambda_{n}(W)\right|} .
$$

The result follows by taking the supremum over all $k$.

Remark 5.5. Results in Lovász and Plummer [19, p. 218] imply that a graph has a nonnegative regular weight matrix if and only if it contains a perfect 2-factor, that is, a spanning subgraph each of whose components is either a cycle or an edge.

As observed earlier, there is no loss in (5.2) if the entries of the nonnegative regular weight matrix $W$ are assumed to be constant on edge orbits in $G$. In particular, if $G$ is edge-transitive and has a regular weight matrix $W$, then $G$ is regular and there is no loss in taking $W=A$, the adjacency matrix of $G$.

The Cartesian product, $G \square H$, of graphs $G$ and $H$ is the graph with vertex set $V(G) \times V(H)$ where vertices $(i, j),\left(i^{\prime}, j^{\prime}\right)$ are adjacent if and only if $j=j^{\prime}$ and $i$ is adjacent to $i^{\prime}$ in $G$, or $i=i^{\prime}$ and $j$ is adjacent to $j^{\prime}$ in $H$. It is not helpful to apply weighted ratio bounds to iterated Cartesian products because the independence ratio $i\left(G^{\square k}\right)=\alpha\left(G^{\square k}\right) / n^{k}$ is monotone decreasing. The limit, $I_{\square}(G)=\inf _{k} i\left(G^{\square k}\right)$, was introduced in [16].

This study would be incomplete without mentioning a completely different type of weighted matrix eigenvalue bound on the independence number, the inertia bound. Unlike the ratio bounds, the inertia bound depends only on the signs of the eigenvalues of a weight matrix.

6. The inertia bound. For an $n \times n$ real matrix $M$, a subspace $U$ of $\mathbb{R}^{n}$ is said to be $M$-isotropic if $x^{\top} M y=0$ for all $x, y \in U$. The Witt index of $M$, denoted by Witt $(M)$, is the maximum of the dimensions of the $M$-isotropic subspaces of $\mathbb{R}^{n}$.

Let $S$ be a maximum independent set of vertices in $G$ and let $U$ be the subspace of vectors $x$ with $x_{i}=0$ when $i \notin S$. Then $U$ has dimension $\alpha(G)=|S|$ and is $W$-isotropic for every weight matrix $W$ of $G$. Thus

$$
\alpha(G) \leq \min _{W} \operatorname{Witt}(W)
$$

where the minimum is taken over all weight matrices $W$ of $G$.

REMARK 6.1. The bound (6.1) still holds when the notions of weight matrix and Witt index are extended to arbitrary fields. When the field is finite, if the bound is attained by a graph $G$, then $n-1-t(G) / 2 \leq \alpha(G) \leq n-t(G) / 2$ where $t(G)$ is the term rank of the adjacency matrix of $G[8$, p. 25]. Thus, the bound is rarely 
attained when the field is finite. Still, the graphs for which equality is attained can be described when the field is finite. They include, for example, graphs $G$ with the König property, $\alpha(G)+\nu(G)=n$, where $\nu(G)$ is the number of edges in a maximum matching $[8$, p. 27, 34, 37].

If $W$ is a weight matrix of $G$, it follows from (6.1) and the spectral theorem for real symmetric matrices that

$$
\alpha(G) \leq \operatorname{Witt}(W)=n_{0}(W)+\min \left\{n_{+}(W), n_{-}(W)\right\}
$$

where $n_{+}(W), n_{-}(W), n_{0}(W)$ denote the numbers of positive, negative and zero eigenvalues of $W$, respectively.

For the case where $W$ is the adjacency matrix of $G$, the bound (6.2) appears in the thesis of Cvetković [4] (see also [5, p. 88]), so (6.2) is often called the Cvetkovic bound. Because the triple $\left(n_{+}(W), n_{-}(W), n_{0}(W)\right)$ is the inertia of $W$, the bound (6.2) is also called the inertia bound.

The inertia bound extends to ordered fields [8, p. 42]. However, as the following question indicates, it is not even known if it is always attained in the real case.

Question 6.2. Does each graph $G$ have a real weight matrix $W$ such that $\alpha(G)=n_{0}(W)+\min \left\{n_{+}(W), n_{-}(W)\right\} ?$

If the answer is positive, then it should be possible to show that there is always a weight matrix for which the inertia bound is better than every weighted ratio bound. Unfortunately, this has proved to be a very difficult task, because the weight matrices that work best for the inertia bound can be quite different from those that work best for weighted ratio bounds.

Still, some progress has been made. In [8], it is proved that it is sufficient to answer Question 6.2 for connected graphs that are $\alpha$-edge-critical with minimum degree at least 3 and having no inclusion between closed neighborhoods. Using results from [19] and a computer search, it turns out that there are only two such graphs when $n \leq 8$ : the graph $K_{2}$ on 2 vertices and the 4-regular graph on eight vertices with $i$ adjacent to $i \pm 1$ and $i \pm 2(\bmod 8)$. It then follows that the answer is positive for all graphs of order $n \leq 8$.

A computer search using a program of B. McKay for generating graphs shows that the answer is also positive for all graphs of order $n \leq 10$. The vertex transitive graphs of order $n \leq 12$ also attain the inertia bound. The Paley graph on $n=13$ vertices is a vertex transitive graph for which the answer to Question 6.2 remains undecided. 
Acknowledgement. The authors are grateful to C. Tardif for his support and helpful conversations.

\section{REFERENCES}

[1] N. Alon, I. Dinur, E. Friedgut, and B. Sudakov. Graph products, Fourier analysis and spectral techniques. Geometric and Functional Analysis, 14:913-940, 2004.

[2] J. I. Brown, R. J. Nowakowski, and D. Rall. The ultimate categorical independence ratio of a graph. SIAM Journal of Discrete Mathematics, 9:290-300, 1996.

[3] Y. Caro. New results on the independence number. Technical Report, Tel-Aviv University, 1979.

[4] D. M. Cvetković. Inequalities based on the basis of the spectrum of the graph. Studia Scientiarum Mathematicorum Hungarica, 8:433-436, 1973.

[5] D. M. Cvetković, M. Doob, and H. Sachs. Spectra of Graphs. Pure and Applied Mathematics 87. Academic Press, New York, 1979.

[6] E. de Klerk, M. W. Newman, D. V. Pasechnik, and R. Sotirov. On the Lovász $\vartheta$-number of almost regular graphs with application to Erdös-Rényi graphs. Preprint.

[7] P. Delsarte. An Algebraic Approach to the Association Schemes of Coding Theory. Ph.D. Thesis, Université Catholique de Louvain, 1973.

[8] R. J. Elzinga. The Minimum Witt Index of a Graph, Ph.D. Thesis, Queen's University, Kingston, 2007. https://qspace.library.queensu.ca/

[9] D. A. Gregory. Hall conditions for edge-weighted bipartite graphs. Seminar notes. https://qspace.library.queensu.ca

[10] C. D. Godsil and M. W. Newman. Eigenvalue bounds for independent sets. Journal of Combinatorial Theory B, 98: 721-734, 2008.

[11] J. W. Grossman, D. M. Kulkarni, and I. E. Schochetman. On the minors of an incidence matrix and its Smith normal form. Linear Algebra and its Applications, 218:213-224, 1995.

[12] W. H. Haemers, On some problems of Lovász concerning the Shannon capacity of a graph, IEEE Transactions on Information Theory, Vol. IT-25, No.2 (1979) 231-232.

[13] W. H. Haemers, Eigenvalue Methods. Packing and Covering in Combinatorics, Mathematical Center Tracts 106. Mathematisch Centrum, Amsterdam 1979.

[14] W. H. Haemers. Eigenvalue Techniques in Design and Graph Theory. Mathematical Center Tracts 121, Mathematisch Centrum, Amsterdam, 1980.

[15] W. H. Haemers. Interlacing eigenvalues and graphs. Linear Algebra and its Applications, 227228:593-616, 1995.

[16] P. Hell, X. Yu, and H. Zhou. Independence ratios of graph powers. Discrete Mathematics, 127:213-220, 1994

[17] R. Horn and C. R. Johnson. Matrix Analysis. Cambridge University Press, New York, 1985.

[18] L. Lovász. On the Shannon capacity of a graph. IEEE Transactions on Information Theory, Vol. IT-25, 1:1-7, 1979 .

[19] L. Lovász and M. D. Plummer. Matching Theory, Annals of Discrete Mathematics 29, NorthHolland Mathematics Studies 121, Amsterdam, 1986.

[20] C. J. Luz, A characterization of Delsarte's linear programming bound as a ratio bound. Linear Algebra and its Applications, 423:99-108, 2007.

[21] C. J. Luz and D. M. Cardoso. A generalization of the Hoffman-Lovász upper bound on the independence number of a regular graph. Annals of Operations Research, 81:307-319, 1998.

[22] C. J. Luz and A. Schrijver. A convex quadratic characterization of the Lovász theta number. SIAM Journal of Discrete Mathematics, 19:382-387, 2005.

[23] M. W. Newman. Independent Sets and Eigenspaces. Ph.D. Thesis, Univ. of Waterloo, 2004.

[24] A. L. Peressini, F. E. Sullivan, and J. J. Uhl. The Mathematics of Nonlinear Programming, 
Springer Undergraduate Texts in Mathematics, 1980.

[25] H. Sachs. Über Teiler, Faktoren und charakteristische Polynome von Graphen, Teil II, Wissenschaftliche Zeitschrift der Technischen Hochschule Ilmenau, 13:405-412, 1967.

[26] J. B. Shearer. The independence number of dense graphs with large odd girth. The Electronic Journal of Combinatorics, 2 (1995) N2.

[27] A. Schrijver. A comparison of the Delsarte and Lovász bounds. IEEE Transactions on Information Theory, Vol. IT-25(4):425-429, 1974.

[28] C. E. Shannon. The zero-error capacity of a noisy channel. IRE Transactions on Information Theory, Vol IT-3:3-15, 1956.

[29] V. K. Wei. A lower bound on the stability number of a simple graph. Bell Laboratories Technical Memorandum, No. 81-11217-9, 1981.

7. Appendix. A quadratic programming problem. The proof of the following lemma is a straightforward extension of that given in [21, p. 310] for the case where $a=1, Q=\frac{A}{\left|\lambda_{n}(A)\right|}+I$ and $A$ is an adjacency matrix.

Lemma 7.1. Let $Q$ be an $n \times n$ positive semidefinite symmetric matrix with eigenvalues $\lambda_{1} \geq \lambda_{2} \geq \cdots \geq \lambda_{m}>0=\lambda_{m+1}=\cdots=\lambda_{n}$. Let $D=\operatorname{diag}\left(\lambda_{1}, \lambda_{2}, \cdots \lambda_{m}\right)$ be the $m \times m$ diagonal matrix of positive eigenvalues of $Q$ and let $U=\left[\begin{array}{llll}u_{1} & u_{2} & \cdots & u_{m}\end{array}\right]$ be an $n \times m$ matrix of corresponding orthonormal column eigenvectors. Let $a \in \mathbb{R}^{n}$.

$$
\begin{aligned}
& \text { If } Q x>a \text { for some } x \in \mathbb{R}^{n} \text { (equivalently, if } U z>a \text { for some } z \in \mathbb{R}^{m} \text { ), then } \\
& \max \left\{2 a^{\top} x-x^{\top} Q x: x \in \mathbb{R}^{n}, x \geq 0\right\}=\min \left\{z^{\top} D^{-1} z: z \in \mathbb{R}^{m}, U z \geq a\right\} .
\end{aligned}
$$

Proof. Since the zero eigenvalues of $Q$ do not contribute to its spectral resolution, $Q=\sum_{i=1}^{m} \lambda_{i} u_{i} u_{i}^{\top}=U D U^{\top}$. Also, because the columns of $U$ are linearly independent, $\operatorname{rank} U=m=\operatorname{rank} Q$ so $Q$ and $U$ have the same range. Thus, $Q x>a$ for some $x \in \mathbb{R}^{n}$ if and only if $U z>a$ for some $z \in \mathbb{R}^{m}$.

Let $(\mathrm{P})$ denote the minimum problem in (7.1). Then $(\mathrm{P})$ is a convex problem and the Lagrangian [24, p. 182] of $(\mathrm{P})$ is

$$
L(z, x)=z^{\top} D^{-1} z+(a-U z)^{\top} x, \text { where } z \in \mathbb{R}^{m}, x \in \mathbb{R}^{n}, x \geq 0 .
$$

The Lagrangian dual $[24$, p. 199] of the minimum problem $(\mathrm{P})$ is the maximum problem

$$
\max _{x \geq 0} h(x) \text { where } h(x)=\min _{z \in \mathbb{R}^{m}} L(z, x) .
$$

For each $x, L(z, x)$ is an unconstrained continuously differentiable strictly convex function of $z$ that is bounded below on $\mathbb{R}^{m}$. Thus, $h(x)=L\left(z^{*}, x\right)$ where $0=$ $\left.\nabla_{z} L(z, x)\right|_{z=z^{*}}=2 D^{-1} z^{*}-U^{\top} x$ or $z^{*}=\frac{1}{2} D U^{\top} x$. Substituting $z^{*}$ for $z$ in $L(z, x)$ 
gives

$$
\max _{x \geq 0} h(x)=\max _{x \geq 0}\left\{a^{\top} x-\frac{1}{4} x^{\top} Q x\right\}=\max _{x \geq 0}\left\{2 a^{\top} x-x^{\top} Q x\right\} .
$$

Because there is a vector $z \in \mathbb{R}^{m}$ such that $U z>a$, the convex problem $(\mathrm{P})$ is superconsistent $[24$, p. 169]. Thus, there is no duality gap [24, p. 210] and equality (7.1) follows. $\square$ 